\title{
Development of Deployment System for Small Size Solar Sail Mission
}

\author{
By Osamu MORI, ${ }^{1)}$ Hirotaka SAWADA,,${ }^{1)}$ Fuminori HANAOKA,${ }^{2)}$ Junichiro KAWAGUCHI, ${ }^{1)}$ \\ Yoji SHIRASAWA, ${ }^{2)}$ Masayuki SUGITA, ${ }^{3)}$ Yasuyuki MIYAZAKI, ${ }^{4)}$ Hiraku SAKAMOTO ${ }^{5)}$ and Ryu FUNASE ${ }^{1)}$ \\ ${ }^{1)}$ JAXA Space Exploration Center, Japan Aerospace Exploration Agency, Sagamihara, Japan \\ ${ }^{2)}$ Department of Aeronautics and Astronautics, The University of Tokyo, Tokyo, Japan \\ ${ }^{3)}$ Department of Mechanical Engineering, Aoyama Gakuin University, Sagamihara, Japan \\ ${ }^{4)}$ Department of Aerospace Engineering, Nihon University, Funabashi, Japan \\ ${ }^{5)}$ Department of Mechanical and Aerospace Engineering, Tokyo Institute of Technology, Tokyo, Japan \\ (Received May 2nd, 2008)
}

The Japan Aerospace Exploration Agency (JAXA) is studying the feasibility of using the solar power sail as a new propulsion engine for deep space exploration missions. In this paper, the sail shape and equipment layout for missions utilizing small-sized solar power sails are proposed. The two-stage deployment method of the sail is also proposed. The sail need to be deployed statically at the first stage, and two types of deployment mechanisms are introduced. On the other hand the second stage of the deployment can be performed dynamically, and the oscillating motion of the membrane is converged by tethers connecting the membrane to the main body. The deployment motions are analyzed by numerical simulations using multi-particle models in order to verify the deployment. They are compared with the results calculated by finite element method models. The numerical simulation results are discussed from the technological viewpoint of the sail deployment dynamics and mechanisms.

Key Words: Solar Sail, Deployment System, Multi-particle Model

\section{Introduction}

The solar sail is a propulsion engine that uses no fuel because it can receive photon momentum. The Japan Aerospace Exploration Agency (JAXA) proposes the use of the solar power sail as a new propulsion engine for deep space exploration missions. It can be a hybrid engine if the ion-propulsion engines, whose specific impulse is very high, are driven by the solar cells on the membrane.

JAXA is studying two missions to evaluate the performance of the solar power sails shown in Fig. 1. The first mission will take place in the early 2010s, and it will involve less technical and financial risk than the second mission. The small-sized solar power sail has been selected for this mission since it is the least costly alternative. The minimum success criteria for this sail are the trouble-free deployment of the sail whose diagonal distance is $20 \mathrm{~m}(\phi 20 \mathrm{~m}$ sail), and the reliability of the electric power supply that uses the solar cells on the membrane. The full success criteria for the sail are the achievement of satisfactory acceleration and navigation on the first use of the photon sail. The second mission will take place in the late $2010 \mathrm{~s}$. It will involve the medium-sized solar power sail having a diameter of $50 \mathrm{~m}$ ( $\phi 50 \mathrm{~m}$ sail), and integrated with ion-propulsion engines. The destinations of the spacecraft will be Jupiter and the Trojan asteroids.

Some kinds of deployment methods have been investigated. $^{1,2)}$ JAXA is studying the spinning type, in which the membrane is deployed and kept flat by the centrifugal force. This deployment method is expected to be accomplished with simpler and lighter-weight mechanisms than other methods because it does not require rigid structural elements. The authors of this paper have been demonstrating this method. The dynamic deployment of a $\phi 10 \mathrm{~m}$ sail was performed successfully using an S-310 sounding rocket on 08/2004. ${ }^{3)}$ The static deployment of a $\phi 20 \mathrm{~m}$ sail was demonstrated using a high altitude balloon on $08 / 2006{ }^{4 \text { ) }}$

In the first half of this paper, the sail shape and equipment layout for missions utilizing small-sized solar power sails are proposed. The two-stage deployment method of the sail is introduced. The sail need to be deployed statically at the first stage, and two types of deployment mechanisms for continuous deployment and divided deployment are also introduced. The second stage of the deployment can be performed dynamically.

In the second half of this paper, the deployment motions are analyzed by numerical simulations in order to verify the deployment. The multi-particle models and FEM (finite element method) models for the deployment analysis of first and second stages are shown respectively. The motions calculated by the multi-particle models are compared with those calculated by the FEM models. The feasibilities of continuous deployment, divided deployment at the first stage and dynamic deployment at the second stage are examined by the numerical simulations considering the initial nutation, tilt angle and mass center offset of the main body and damping of the tether connecting the membrane to the main body. 


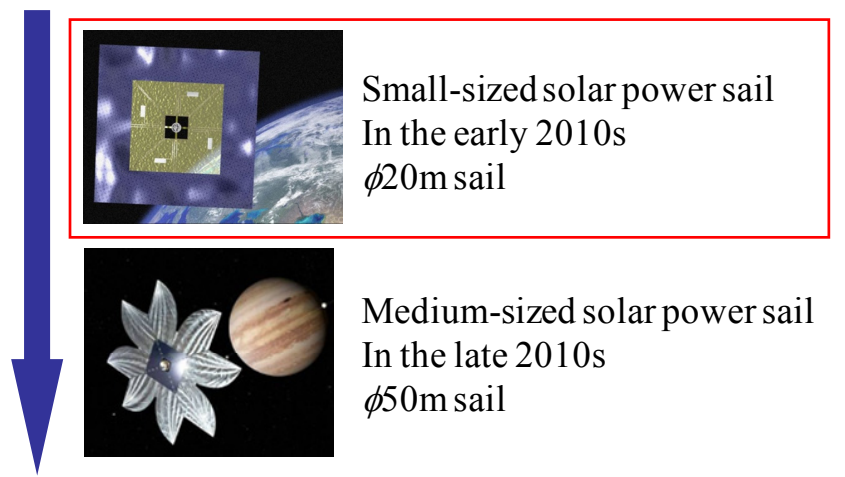

Fig. 1. Solar power sail missions

\section{Proposals for the Small-Sized Solar Power Sail}

\subsection{The sail}

The proposed sail shape and equipment layout is shown in Fig. 2.

Membrane: The shape of the membrane is a square whose diagonal distance is $20 \mathrm{~m}$. It consists of four trapezoid petals. They are connected with one another by bridges. The direction of folded lines is perpendicular to the direction of the centrifugal force. It is made of polyimide whose thickness is $7.5 \mu \mathrm{m}$. It is relatively easy to produce. Tether: The membrane should not contact the main body after the deployment. The membrane is connected to the main body by tethers.

Tip mass: Four masses are attached to the four tips of the membrane. The tip masses adjust the centrifugal force and the inertial momentum of the membrane.

Thin film solar cell: Several a-Si (amorphous silicon) cells are attached to certain areas of the membrane. The area ratio with respect to the membrane is $5 \%$.

Steering device: Some variable reflectance elements are loaded near the tips of the membrane. They can switch specular and diffuse reflection by power on/off. The spin direction can be controlled by the solar pressure torque as shown in Fig. 3. ${ }^{5}$

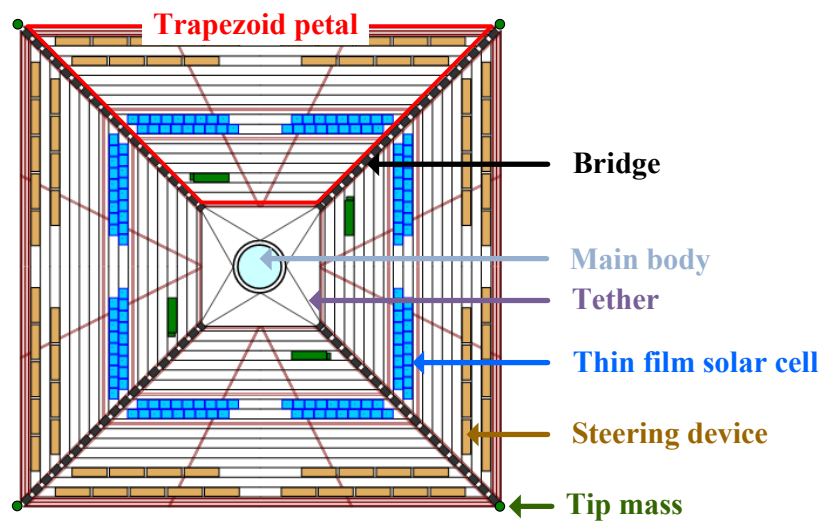

Fig. 2. Sail shape and equipment layout

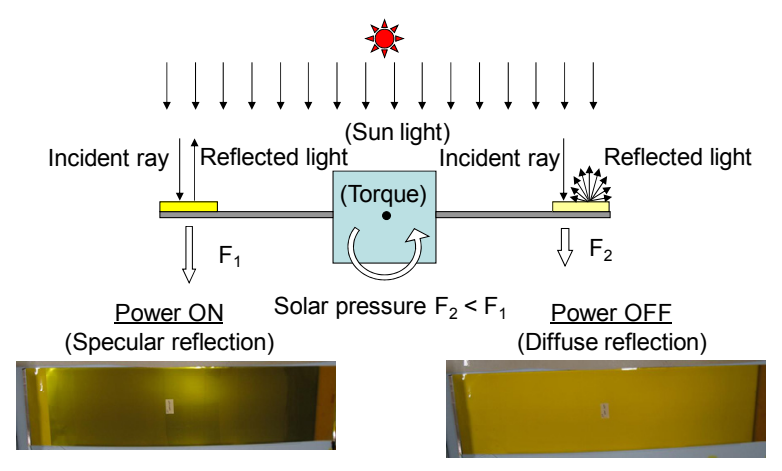

Fig. 3. Steering device

\subsection{Deployment}

The proposed deployment method of the sail is shown in Fig. 4. It consists of two stages. In the folded configuration, each petal is line-shaped and rolled up around the satellite as shown in (1). In the first stage, the rolled petals are extracted like a Yo-Yo despinner, and form a cross shape. The shape is maintained by stoppers as shown in (3). In the second stage, the stoppers are released and each petal expands to form a square shape. If the first stage of the deployment is performed dynamically, each petal would be twisted around the main body just after the deployment. Therefore, the first stage of the deployment needs to be performed statically. On the other hand, the second stage of the deployment can be performed dynamically.

This paper introduces two types of deployment mechanisms. Fig. 5 shows a continuous deployment mechanism. The Yo-Yo despinner is restricted by a stopper. Each stopper slowly rotates relative to the main body to deploy each petal continuously during the first stage of the deployment. The second stage of the deployment is initiated by releasing four stoppers after the first stage of the deployment. Fig. 6 shows a divided deployment mechanism. Each petal is held by several stoppers, which are properly aligned. The petals are deployed step-by-step in the first stage of the deployment by releasing stoppers 1-5 in numerical order. The petals are deployed dynamically in the second stage of the deployment by releasing stopper 6 . This mechanism is relatively simple; however, the deployment sequence in the first stage is complicated.

The deployment sequence is defined as follows:

1) Separation from rocket with slow spin $(0.21 \mathrm{rad} / \mathrm{s})$

2) Release of launch lock

3) Spin up using RCS (0.21 rad/s -> 3.77 $\mathrm{rad} / \mathrm{s})$

4) First stage of the deployment (3.77 rad/s -> 1.33rad/s)

5) Spin down using RCS (1.33 rad/s -> $0.81 \mathrm{rad} / \mathrm{s})$

6) Second stage of the deployment $(0.81 \mathrm{rad} / \mathrm{s} \quad->$ $0.32 \mathrm{rad} / \mathrm{s}$ )

7) Spin down using RCS (0.32 rad/s ->0.10rad/s)

8) Control of spin direction using RCS and steering devices

The spin rate is decreased in the first and second stages of the deployment because the inertial momentum of the sail is increased. 


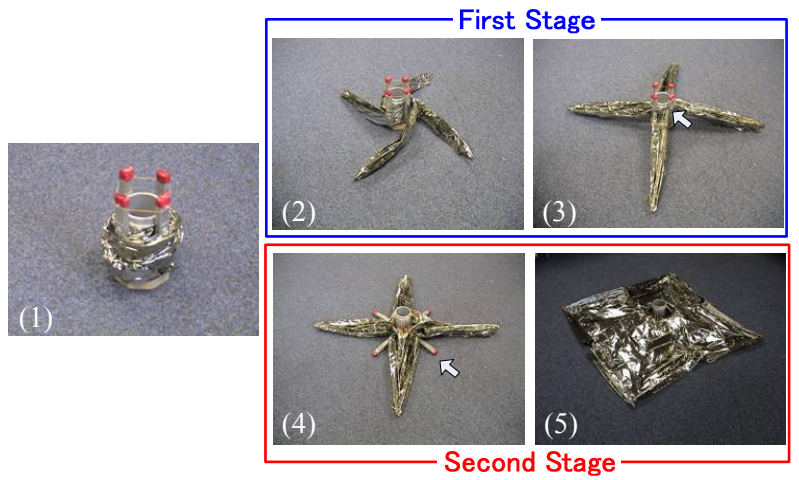

Fig. 4. Deployment method

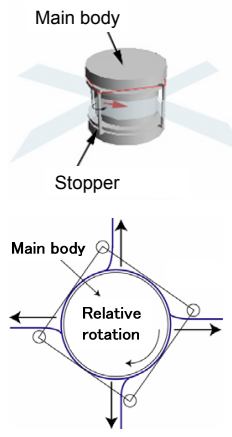

(1) First stage

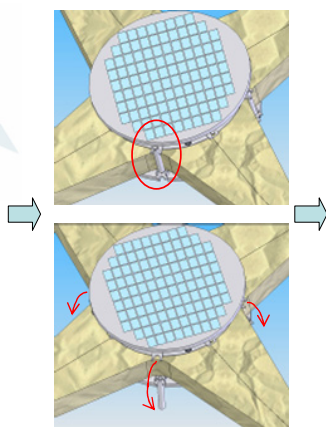

(2) Stopper release

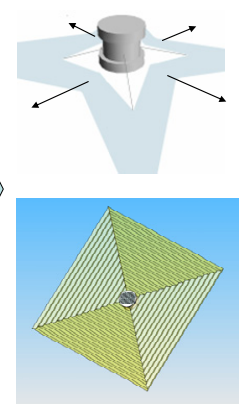

(3) Second stage
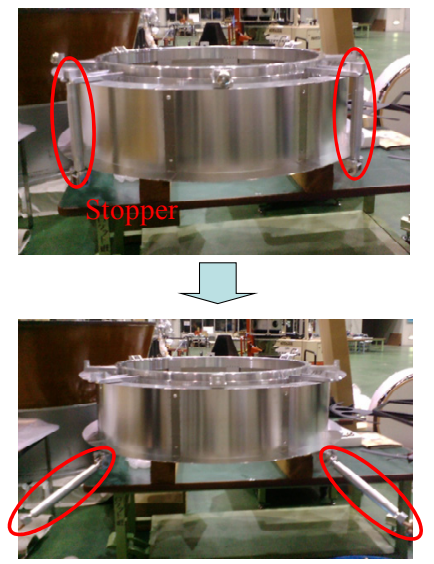

Fig. 5. Continuous deployment mechanism
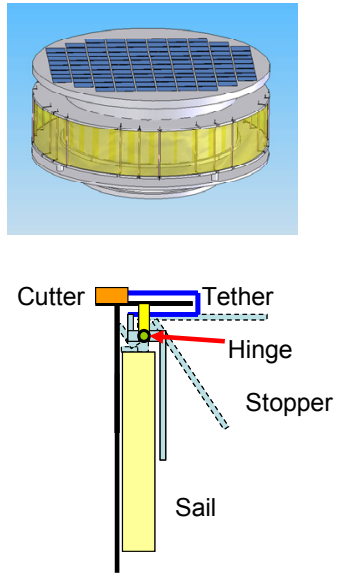

(1) Folded sail

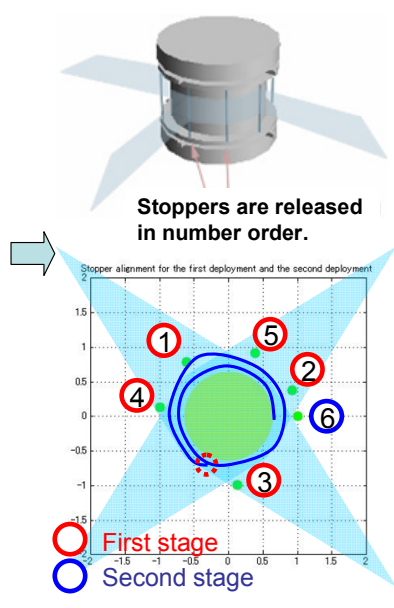

(2) Stopper release
Fig. 6. Divided deployment mechanism

\section{Deployment Motion Analysis by Numerical Simulations}

\subsection{Mass property}

In this chapter, the deployment motion of the sail is verified by numerical simulations. The mass property of the main body and the sail are set as follows:

$<$ Main body $>$

Weight: $300 \mathrm{~kg}$

Inertial moment:

$\left\{I_{x x}, I_{y y}, I_{z z}, I_{x y}, I_{y z}, I_{z x}\right\}=\{53.9,46.7,80.9,1,-0.1,0.8\}\left[\mathrm{kgm}^{2}\right\rfloor$

Mass center offset: $0.02 \mathrm{~m}$ (-z axis)

$<$ Sail $>$

Weight of membrane: $3.75 \mathrm{~kg} * 4$

Weight of tip mass: $0.5 \mathrm{~kg} * 4$

Weight of tether: $0.02 \mathrm{~kg} * 16$

Young's modulus of membrane: $3.2 \mathrm{GPa}$

Young's modulus of tether: $60 \mathrm{GPa}$

Damping coefficient of membrane: 0

Damping coefficient of tether: 0.0000002

Poisson's ratio of membrane: 0.3

\subsection{Sail models of first stage of the deployment}

Analytical model of the first stage of the deployment are shown in Fig. 7.

$<$ Main body $>$

Shape: cylinder

Diameter: $1.26 \mathrm{~m}$

Height: $0.8 \mathrm{~m}$

$<$ Sail $>$

Shape: rectangle

Height: $0.30 \mathrm{~m}$

Wide: $6.5 \mathrm{~m}$

The motions of four petals are independent of one another.

There are two sail models. In multi-particle model, each petal consists of 32 particles and the tip mass is assumed as a particle. These particles are assumed to be connected by springs. The spring constants are derived by the principle of virtual work, so as to rationally satisfy the relations of displacement energy. The damping coefficient of the membrane is equal to zero. If the membrane is slacked $\left(L<L_{0} ; L\right.$ and $L_{0}$ are the actual and base distances between two particles, respectively), the tension becomes zero.

In FEM model, each branch is modeled by the finite Timoshenko beam elements with variable element length. The axial, bending, transverse-shear and torsional stiffness of each branch are taken into account in this model. The number of the beam element is fixed during the first stage of the deployment. The unstressed length of each beam element elongates gradually, so that the length of the branch is extended during the deployment.

The difference between these models is that the FEM model can consider the effect of the bending of the branch while the multi-particle model cannot.

There is another difference. The multi-particle model employs the explicit Runge-Kutta-Gill scheme for 
numerical time integration. In the simulations of multi-particle model, the time stepping size is enough small to converge the deployment motion. The FEM model employs the energy-momentum method. ${ }^{6,7)}$ In all simulations of multi-particle and FEM models, it is confirmed that the linear and angular momentums are conserved.

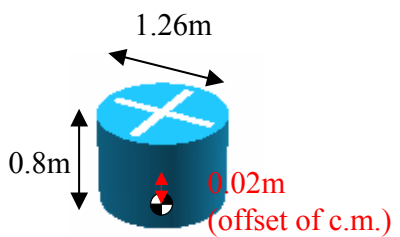

(a) Rigid cylinder model of the main body

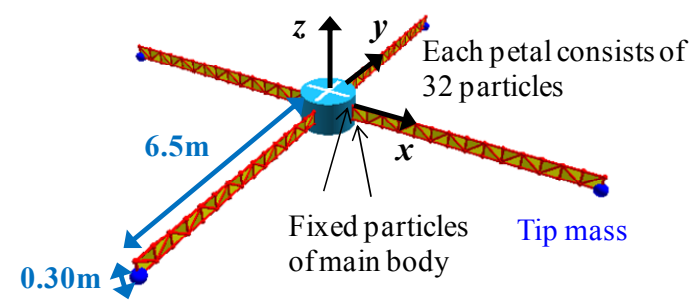

Membrane tension

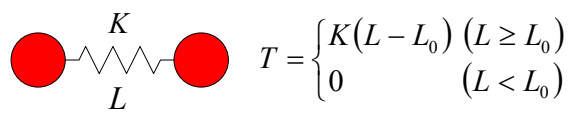

(b) Multi-particle model of the sail

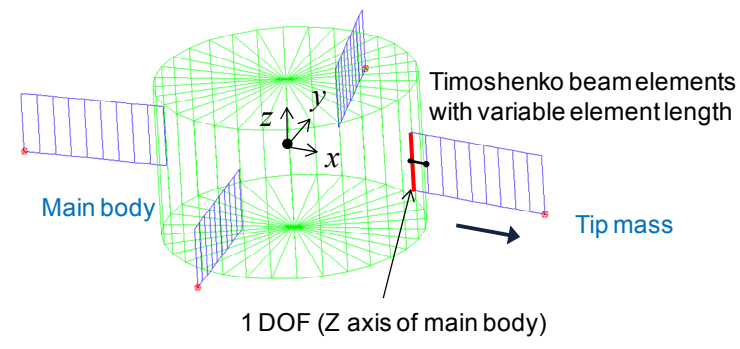

(c) FEM model of the sail

Fig. 7. Analytical model of the first stage deployment

\subsection{Results of first stage of the deployment}

Considering the deployment sequence, the initial conditions of numerical simulation for the first stage of the deployment are set as follows:

Initial spin rate: $3.77 \mathrm{rad} / \mathrm{s}$

Initial nutation angle: $5 \mathrm{deg}$

Fig. 8 shows pictures of the continuous deployment motion using multi-particle model. In this case, it is supposed that each petal is deployed in 120 seconds at a constant speed.

Fig. 9 shows the graphs of spin rate, attitude angle and tip angles of the first stage. Tip angles (in-plane and out-of-plane) are defined in Fig. 10.
Fig. 9 (a) shows the continuous deployment motion using multi-particle model. The spin rate is decreased because the inertial momentum of the sail is increased during the continuous deployment $(0-120 \mathrm{sec})$, and it becomes constant after the deployment. The attitude angle and tip angles (in-plane and out-of-plane) are oscillating. They are not converged because the membrane damping is zero. The tip angle (in-plane) is increased just after the continuous deployment $(120 \mathrm{sec})$. It is, however, not more than $20 \mathrm{deg}(<45 \mathrm{deg})$. Thus each stopper does not collide with each petal if it is released as shown in Fig. 5 .

Fig. 9 (b) shows the continuous deployment motion using FEM model. The spin rate, attitude angle calculated by FEM model are nearly equal to those calculated by multi-particle model, respectively. The tip angles (in-plane and out-of-plane) become smaller due to bending stiffness. However, the effect of bending stiffness on the continuous deployment motion is little, because it is enough small. If it becomes larger, the vibration of tip angle (in-plane) becomes smaller.

Fig. 9 (c) shows the divided deployment motion using multi-particle model. In the case that the deployment is divided into 5 and that stoppers are released every 800 seconds. The spin rate, attitude angle and tip angles (in-plane and out-of-plane) are oscillating drastically just after every stopper is released. There is a possibility that the petals collide with each other, since the maximum amplitude of the tip angle (in-plane) is 120deg. The vibration amplitudes can be decreased if the division number is increased or dampers are included. The deployment mechanism becomes, however, complicated.

These results show that the continuous deployment at the first stage is feasible in the condition that the initial nutation, tilt angle, mass center offset of the main body are not zero and the membrane damping is zero. On the other hand, the feasibility of the divided deployment is dependent on the division number and dampers.

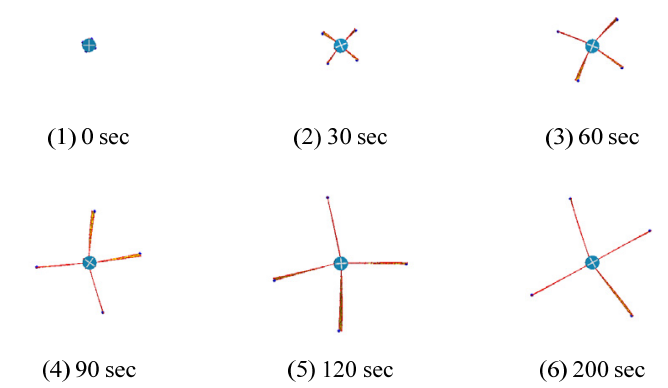

Fig. 8. Pictures of continuous deployment motion using multi-particle model 

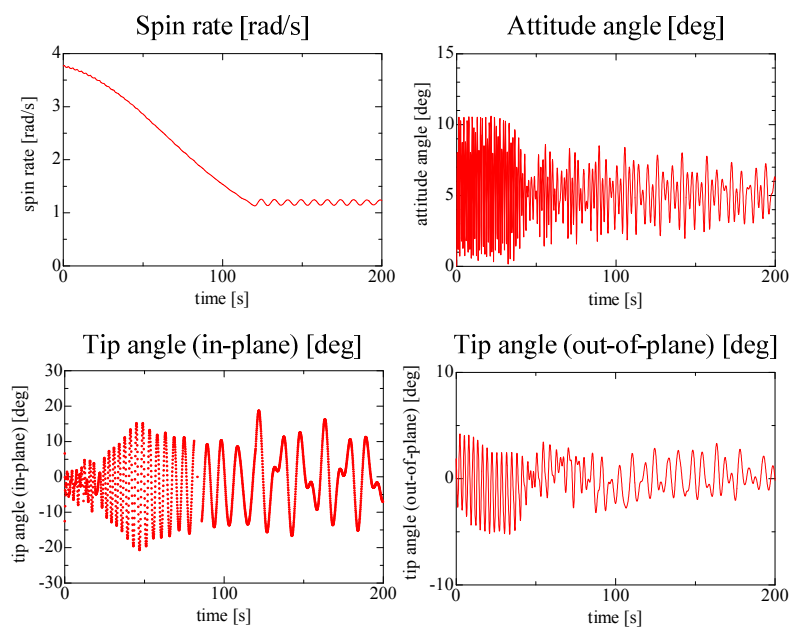

(a) Continuous deployment using multi-particle model
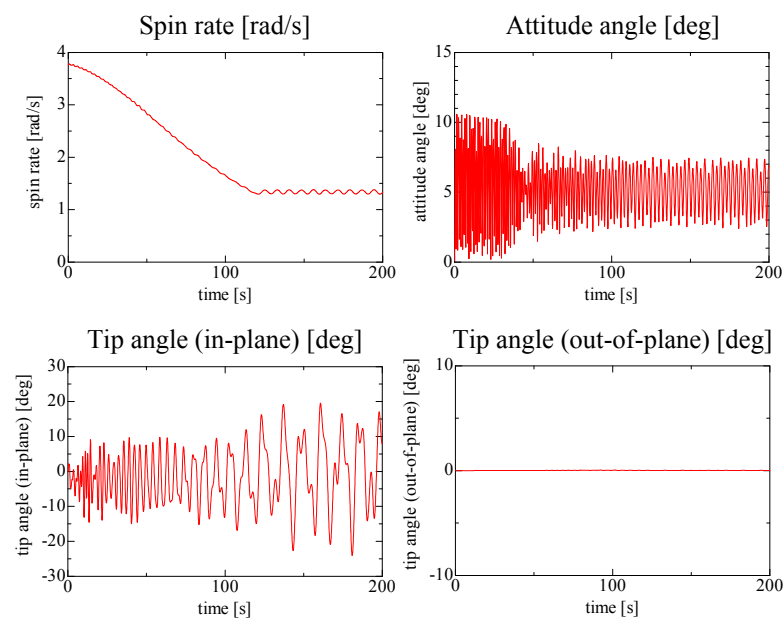

(b) Continuous deployment using FEM model
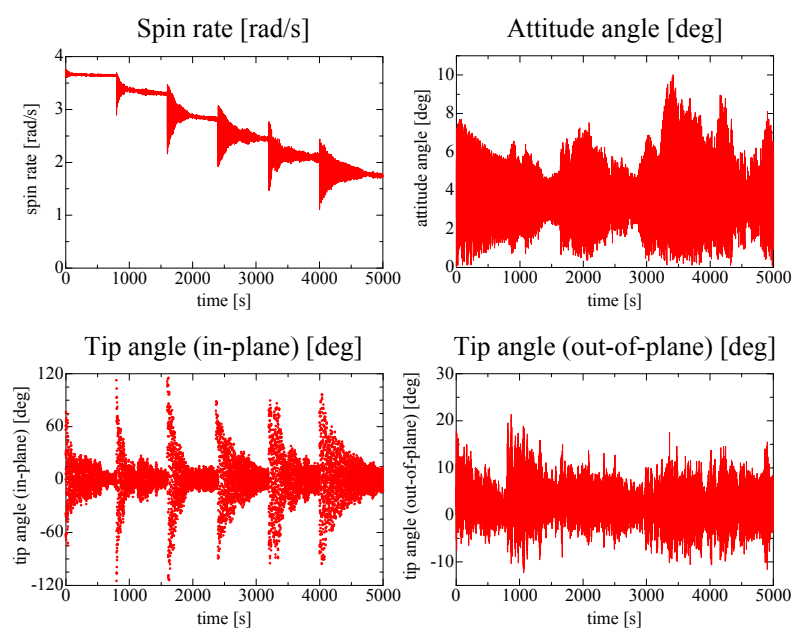

(c) Divided deployment using multi-particle model

Fig. 9. Motions of first stage deployment

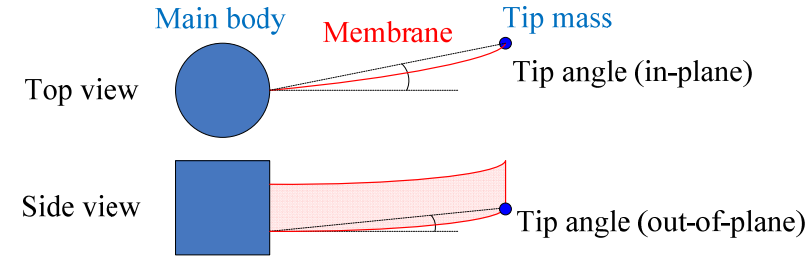

Fig. 10. Definition of tip angles of first stage deployment

\subsection{Modeling of second stage of the deployment}

Analytical model of the second stage of the deployment are shown in Fig. 11. The model of the main body is the same as the first stage of the deployment.

$<$ Sail $>$

Shape: square

Inside diagonal distance: $4.5 \mathrm{~m}$

Outside diagonal distance: $20 \mathrm{~m}$

Bridge length: $0.015 \mathrm{~m}$

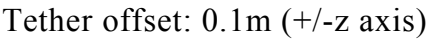

Four petals are connected with one another by bridges. The membrane is connected with the main body by 16 tethers.

There are two sail models. In multi-particle model, each petal consists of 134 particles and the tip mass is assumed as a particle. The density of the membrane is constant. These particles are assumed to be connected by springs. Each tether consists of 4 particles. The particles of the tether are assumed to be connected by springs and dampers. The damping coefficient of the tether is enough small, but not zero. If the tether is slacked, the tension becomes zero.

The FEM model is the finite element plane stress model based on the tension field theory. The membrane is assumed to be isotropic and to be unable to bear compressive stress. At the moment that the principal stress gets negative, the stress is set to be zero.

The essential difference between the multi-particle model and the FEM model is as follows; The multi-particle model assumes that the stress in the direction along each spring depends only on the strain in the same direction, so that the elasticity matrix (stress-strain relation matrix) is approximated to be diagonal. On the other hand, the FEM model can take into account the effect of the strain in the orthogonal direction because it is the plane stress model. ${ }^{8)}$

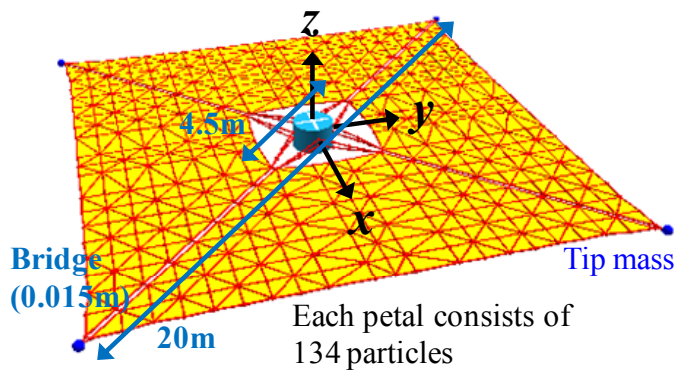

Fig. 11. Analytical model of second stage deployment (continued to the next page) 


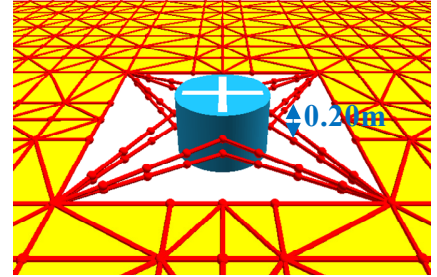

Each tether consists of 4 particles

Tether tension

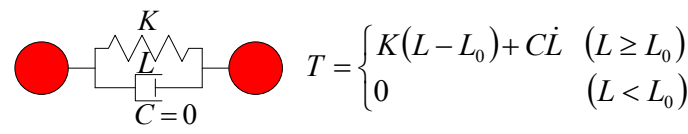

(a) Multi-particle model of the sail
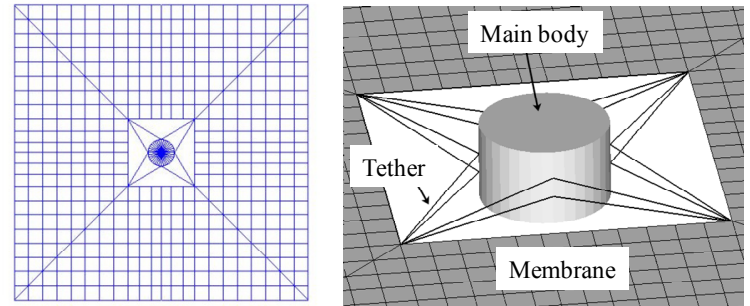

(b) FEM model of the sail

Fig. 11. Analytical model of second stage deployment

\subsection{Results of second stage of the deployment}

Considering the deployment sequence, the initial conditions of numerical simulation for the second stage of the deployment are set as follows:

Initial spin rate: $0.81 \mathrm{rad} / \mathrm{s}$

Initial nutation angle: $5 \mathrm{deg}$

Fig. 12 shows pictures of the nominal motion using multi-particle model. The second stage of the deployment is conducted dynamically. The sail is expanded dynamically in a few seconds. After that, it does not collide with the main body.

Fig. 13 shows the graphs of spin rate, attitude angle and tip angles of the second stage. Tip angles (in-plane and out-of-plane) are defined in Fig. 14.

Fig. 13 (a) shows the nominal motion using multi-particle model. The spin rate, attitude angle and tip angles (in-plane and out-of-plane) are changed dramatically just after the deployment start. The vibrations of spin rate and tip angles (in-plane) are converged by tether damping. On the other hand, the attitude angle and tip angle (out-of-plane) are not converged because the tilt angle (product of inertia) of the main body is not zero and the membrane damping is zero.

Fig. 13 (b) shows the nominal motion using FEM model. The spin rate, attitude angle and tip angles (in-plane and out-of-plane) calculated by FEM model are nearly equal to those calculated by multi-particle model, respectively.

Fig. 13 (c) shows the motion in the case of no tilt angle (product of inertia) of main body using multi-particle model. The attitude angle is decreased. The average of tip angle (out-of-plane) is zero. They are converged to zero, if the membrane damping is not zero.

Fig. 13 (d) shows the motion in the case of no mass center offset of the main body using multi-particle model. The attitude angle at the beginning of the deployment is decreased, because the main body is less inclined by the tether tension.

Fig. 13 (e) shows the motion in the case that the connection method using tethers are changed as shown in Fig. 15. The torques (in-plane and out-of-plane) of the main body by the tethers become smaller. Thus the vibrations of spin rate and tip angle (in-plane) are converged more slowly and the attitude angle and tip angle (out-of-plane) becomes larger.

These results show that taking advantage of tether damping, the dynamic deployment at the second stage is feasible in the condition that the initial nutation, tilt angle, mass center offset of the main body are not zero and the membrane damping is zero.

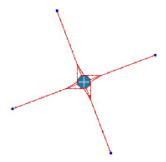

(1) $0 \mathrm{sec}$

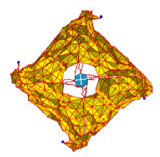

(4) $5 \mathrm{sec}$

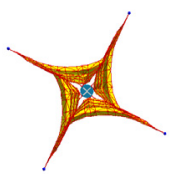

(2) $1 \mathrm{sec}$

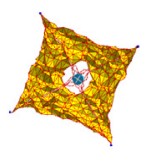

(5) $30 \mathrm{sec}$

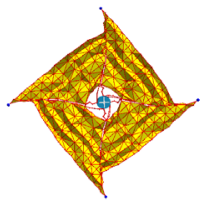

(3) $2 \mathrm{sec}$

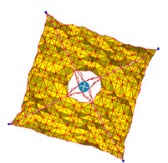

(6) $3000 \mathrm{sec}$
Fig. 12. Pictures of nominal motion using multi-particle model
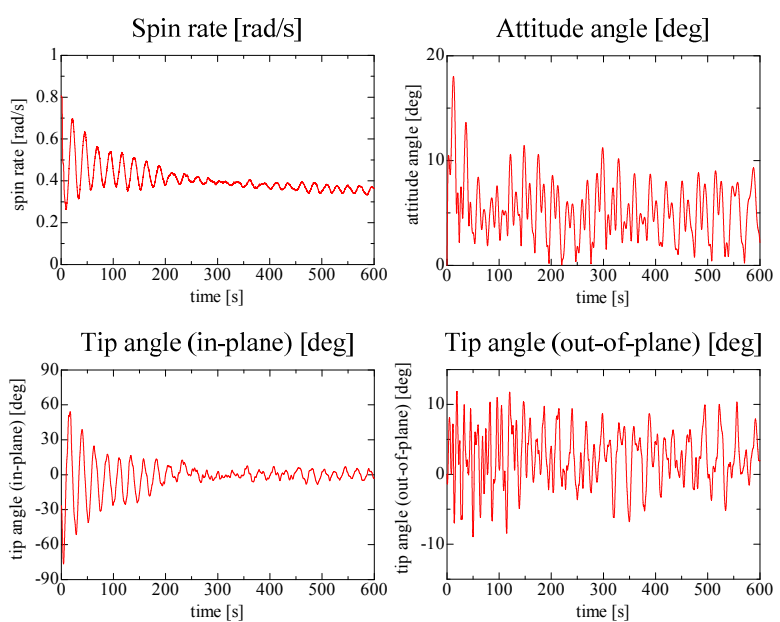

(a) Nominal motion using multi-particle model

Fig. 13. Motions of second stage deployment (continued to the next page) 

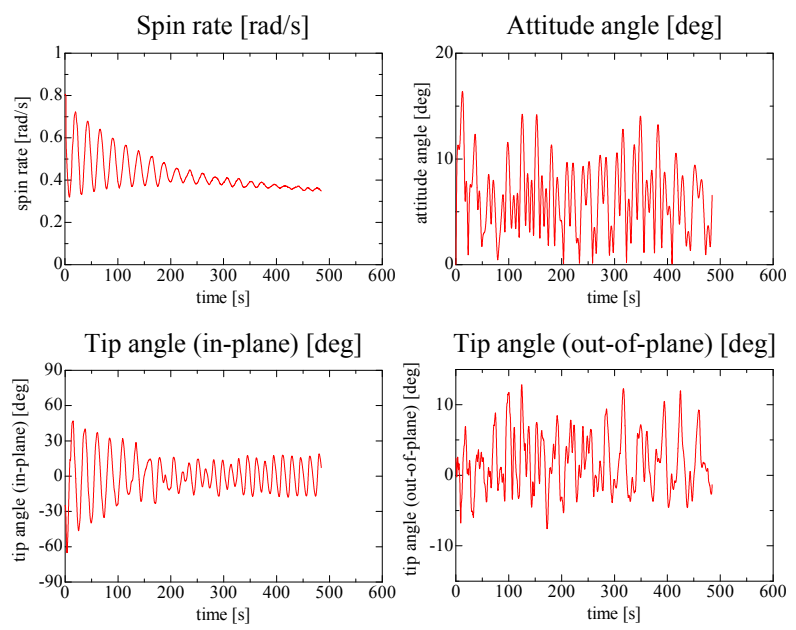

(b) Nominal motion using FEM model
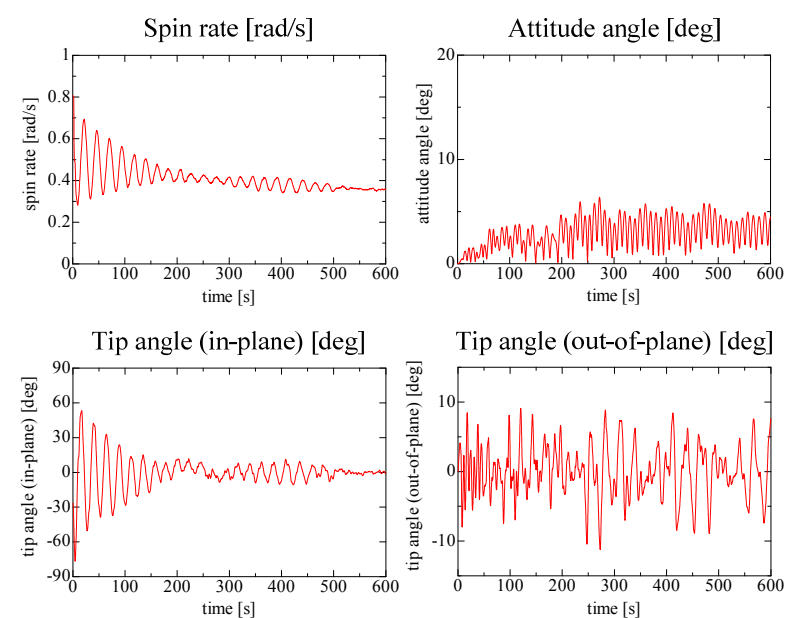

(c) No tilt angle
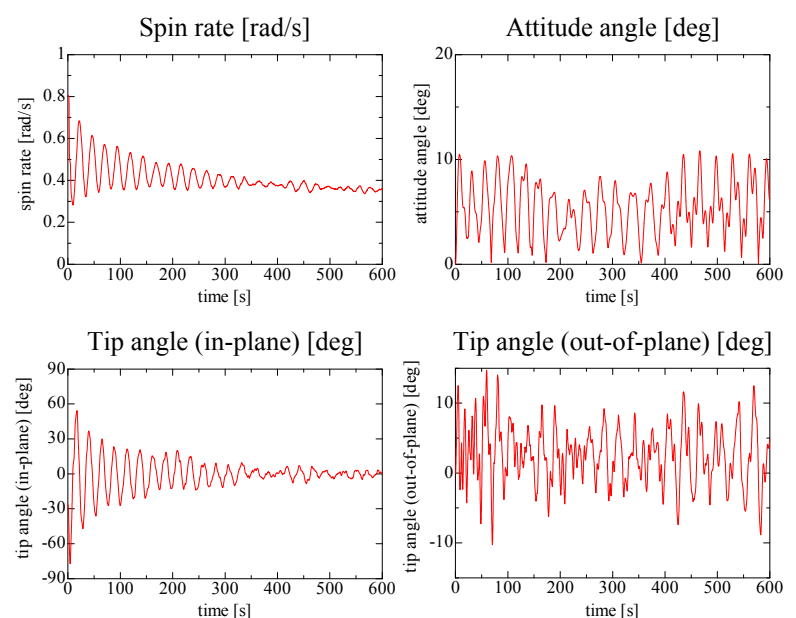

(d) No mass center offset

Fig. 13. Motions of second stage deployment (continued to the next page)
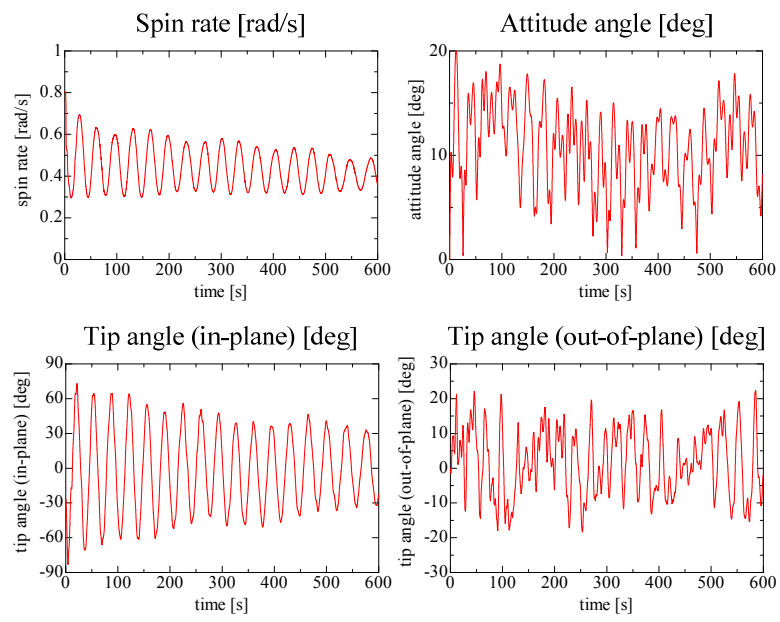

(e) Different connection method using tethers

Fig. 13. Motions of second stage deployment

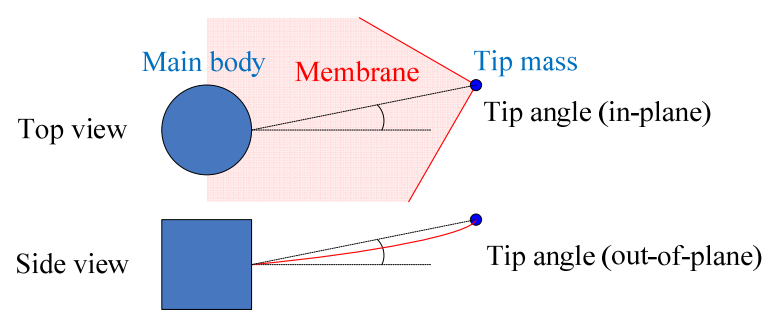

Fig. 14. Definition of tip angles of second stage deployment
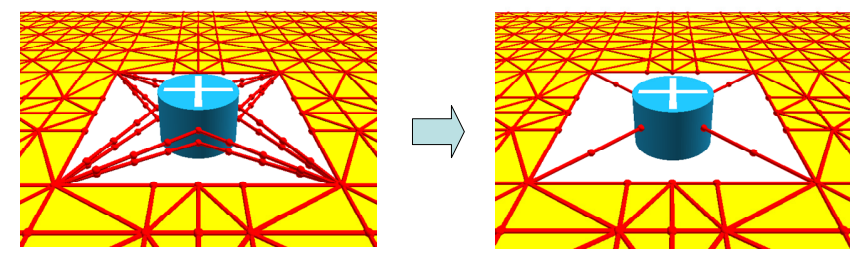

Fig. 15. Connection method using tethers

\section{Conclusions}

For small-sized solar power sail missions, the square-shaped sail and its equipment layout were proposed. The two-stage deployment of the sail was also proposed. The continuous deployment mechanism and divided deployment mechanism were introduced for static deployment at the first stage.

The multi-particle models and FEM models for the deployment of first and second stages were shown respectively. The motions calculated by multi-particle models were well accorded with those calculated by FEM models. The feasibility of continuous deployment at the first stage and dynamic deployment at the second stage were confirmed by the numerical simulations considering the initial nutation, tilt angle, mass center offset of the main body and tether damping. 


\section{References}

1) Greschik, G. and Mikulas, M. M.: Design Study of a Square Solar Sail Architecture, J. of Spacecraft and Rockets, 39 (2002), pp.653-661.

2) Hinkle, J. D., Warren, P. and Peterson, L. D.: Geometric Imperfection Effects in an Elastically Deployable Isogrid Column, J. of Spacecraft and Rockets, 39 (2002), pp.662-668.

3) Tsuda, Y., Mori, O., Takeuchi, S. and Kawaguchi, J.: Flight Result and Analysis of Solar Sail Deployment Experiment using S-310 Sounding Rocket, Space Technol., 26 (2006), pp. 33-39.

4) Nishimaki, S., Mori, O., Shida, M. and Kawaguchi, J.: Stability and Control Response of Spinning Solar Sail-craft containing A Huge Membrane, 57th International Astronautical Congress, IAC-06-C1.1.07, 2006.

5) Hanaoka, F., Mori, O., Funase, R., Tsuda, Y. and Kawaguchi, J.: On the Feasibility of Navigation Control of Solar Sail Spacecraft, Proceedings of 17th Workshop on Astrodynamics and Flight Mechanics, pp.240-244, 2007.

6) Simo, J. C., Posbergh, T. A. and Marsden, J. E.: Stability of Coupled Rigid Body and Geometrically Exact Rods: Block Diagonalization and The Energy-Momentum Method, Physics Reports, 193 (1990), pp.279-362.

7) Miyazaki, Y.: Wrinkle/Slack Model and Finite Element Dynamics of Membrane, International Journal for Numerical Methods in Engineering, 66 (2006), pp.1179-1209.

8) Miyazaki, Y. and Iwai, Y.: Dynamics Model of Solar Sail Membrane, Proceedings of 14th Workshop on Astrodynamics and Flight Mechanics, pp.32-37, 2004. 